\title{
Partial edentulism and Temporomandibular joint disorders
}

\author{
Swati Gupta ${ }^{1}$, Richa Gupta ${ }^{2}$, RajeevGarg ${ }^{3}$ \\ ${ }^{1}$ (MDS, Periodontology, Chandigarh, India) \\ 2 (MD Anatomy, PGIMER/Chandigarh, India) \\ ${ }^{3}$ (MCH Neurosurgery, Fortis hospital/Chandigarh, India)
}

\begin{abstract}
The loss of teeth has serious functional, esthetic disabilities, in addition to compromising the patients' quality of life. Currently, in the private sector, the status of prosthetic rehabilitation is only limited to anterior teeth due to esthetic reasons and rarely the posteriors. This could be called as inefficiency or lack of knowledge on part of few dental practitioners to understand and explain the importance of functional occlusion to the masses. Occlusion and its relationship with the temporpmandibular joint has always been debated. However, there are studies that have tried to elucidate this quite often ignored aspect of rehabilitation. The purpose of this review is to illuminate the relationship between the teeth and temporomandibular joint harmonious relationship. The authors intend to create an awareness and encourage the dentists to conduct long term trials on this controversial yet an important aspect of prosthetic dentistry.
\end{abstract}

Keywords: missing teeth, temporomandibular joint dysfunction, rehabilitation, occlusion

\section{INTRODUCTION}

The stomatognathic system is a specific arrangement of various structures of the oral cavity and facial skeleton working together, in particular its key elements: teeth with the parodontium, masticatory muscles, and temporomandibular joints (TMJ). [1] The temporomandibular joint (TMJ) is considered one of the most complex joints of the body and classified as a compound joint made up of two bones and the disk between the bones, a disk considered functioning like a third bone.[2] Patient comfort and orofacial stability depend on harmonious coordination between these key elements. Temporomandibular disorders (TMD) are defined by the American Academy of Orofacial Pain as "a collective term that embraces a number of clinical problems that involve the masticatory muscles, the TMJ [temporomandibular joint], and the associated structures." [3] The common signs and symptoms of TMDs are:

1. Pain or tenderness in the temporomandibular joint, muscles of mastication, facial areas, ear region, shoulder and neck

2. A clicking, popping or grating sound when opening or closing the mouth or while chewing

3. Catching or locking of the joint with deviations or deflections of the mandible on opening or closing the mouth

4. Limitations in opening or closing the mouth

5. Difficulty or discomfort while chewing

6. Sensation of an uncomfortable bite

The etiology of TMD is multifactorial and these include trauma, genetics, anatomical factors and occlusion. the most popular theories regarding TMD etiology are based on the biopsychosocial model, which involves a combination of biological, psychological and social factors. [4,5] The theories of TMD etiology that have made the largest impact are related to various types of occlusal imperfection. This review focuses on the most important and still the most ignored etiological factor i.e. occlusion.

\section{Occlusion and Temporomandibular joint}

Occlusion embraces the dynamic morphological and functional relationships between all components of the masticatory system - not just teeth and their supporting tissues but also the neuromuscular system, the temporomandibular joints (TMJs) and the craniofacial skeleton.[6,7] Occlusion as one of the causative factors for TMD has always been a matter of debate. In a review of 57 epidemiological studies of the relation between occlusion and TMDs, Okeson found that 35 suggested a relation compared with 22 studies that suggested no relation. The "positive" occlusal findings in the 35 studies varied so widely that no consistent feature could be identified. The occlusal disharmonies cited in these studies were also prevalent among many symptom-free people. [8] On the other hand, in a study role of morphologic and functional occlusal factors with respect to development of TMDs was found weak. [9] A recent study has suggested that the relationship between occlusal treatments of TMD problems is controversial because it is not strictly demonstrated in numerous scientific studies. Occlusion is not the dominant cause of TMD problems. [10]Another systematic review aimed at assessing the association of malocclusion and functional occlusion with temporomandibular disorders has 
concluded that few associations exist between malocclusion and parameters of functional occlusion and clinical as well as subjective TMD, but these associations were not uniform. The occlusal factors found were partly protective for TMD, ie, subjects with these occlusal parameters showed fewer signs and symptoms of TMD (angle Class II malocclusion, deep bite, anterior crossbite). Thus, it was suggested from the results that further large scale studies are required to determine the strength of the association.[11] The question that always arises is, which are the factors that can have an association with TMD, since no occlusion is perfect and malocclusion cannot be considered a pathology that would always create orofacial disturbances. A number of occlusal factors have been examined in an attempt to assess their individual effect on TMD. These have included skeletal anterior open bite, overbite, overjet, crossbite, incisor inclination, missing teeth, posterior occlusal support, balancing-side interferences, working-side interferences, intercuspal interferences, symmetry of contacts in the retruded contact position, and slide between the retruded contact position and the intercuspal position. Of all the occlusal factors, loss of teeth and the impact of edentulousess on instability on the temporomandibular joint functioning cannot be ignored.

\section{Missing anterior teeth and TMD:}

Role of anterior teeth in functional occlusion- In an ideal functional occlusion, the anterior act as a group with the cuspids as the main guiding inclines to gently but immediately disengage or disocclude anterior teeth. The lift or the guidance of the anterior teeth should be in harmony with the pattern that is dictated by the temporomandibular joint. The incisal edges of mandibular teeth occlude with lingual surface of maxillary teeth and the steepness of these lingual surfaces determines the amount of vertical movement of the mandible. Anterior guidance is determined by the vertical and horizontal overlap between anterior teeth. The change in the anterior guidance will have an impact on the posteriors, indirectly affecting the health of the TMJ. Loss of anterior teeth induces posterior interference with extended disocclusion time. Posterior disocclusion is critical to remove the harmful force on the teeth, temporomandibular joint and eliminate muscle hypertonicity. Establishing optimum anterior guidance is a key to establishing harmonious functional occlusion in addition to the correction of the esthetic and phonetic disabilities. [12] In restorative dentistry, clinical experience has demonstrated that a change or modification of unfavorable incisal guidance will have a favorable influence on teeth, restorations and TMJs. [13]

\section{Missing posterior teeth and TMD:}

The factors supporting the hypothesis of an association between posterior teeth and functioning of TMJ, to name a few are

1. The absence of posterior support results in overloading of the temporomandibular joint (TMJ) structures. [14]

2. It has been assumed and experimentally proved that the absence of posterior teeth would result in mandibular overclosure and as a consequence, the condyles would deviate from their normal centric position in the TMJ, causing dislocation in the joint. $[15,16]$

3. Joint pain has also been reported more frequently on the side with most missing teeth and increased risk of joint disorders were found in subjects without any molar support.[17]

4. Correlation between absence of posterior support and osteoarthritis of TMJ was reported in several studies.[18] However, this has been controversial as few other studies have concluded that risk of osteoarthritis of the temporomandibular joint in people without posterior teeth was not different from those with complete dental arches. Furthermore no correlation could be found between the number of remaining teeth and severity of symptoms of temporomandibular disorders. [19,20]

5. Missing posterior teeth has been associated with the TMJ sound like clicking. Previous studies have also observed that TMJ sounds may originate from changes in articular surfaces, deviations in the form of articular components [21,22] and lack of muscle coordination.[23] In another study, it was observed that loss of molar support was involved in the initiation of lesions on the load bearing articular surfaces of the condyle and articular eminence of younger individuals.[24] A recent study examined the relationship between missing mandibular molars and TMJ dysfunction. It was concluded from the study that clicking joint sound and condylar flattening of the TMJ was more significant in unilateral loss of molars as compares to bilateral loss. [25]

\section{Partial lateral edentulism is harmful for TMJ health?}

The lateral teeth, that is, molars and premolars, make up the area of occlusal support and play a decisive role in setting the position of the mandible against the maxilla and so they also have an impact upon the topographical relations and the TMJ function. The absence of molars and premolars makes the other residual teeth in the oral cavity absorb even greater load than before, for which they are not prepared and which may lead to their faster abrasion and lowering of the occlusal height. This results in the mandible coming closer to the maxilla and frequently also the posterior dislocation of the mandible. Such a change of the position of the mandible against the maxilla disrupts the TMJ biomechanics and may generate various temporomandibular 
disorders (TMD), as a result of changes in the spatial relation of the articular disc, fossa and mandibular condyle. If the organism fails to compensate for such dysfunctions, the patient will feel persistent pain in the TMJs, but also the masticatory muscles and various areas across the head. The resulting change in the tension of the paravertebral muscles causes pain radiating to the neck, the shoulder girdle and even the lower spine. $[26,27,28]$ A study axiographically analysed the mobility of the condyles of the temporomandibular joints in patients with partial edentulism in lateral sections and specify whether the partial edentulism has an impact on the severity of temporomandibular disorders. The results showed some correlation between the advancement of temporomandibular joint disorder, the range of partial edentulism and the abrasion of the residual teeth. A significant intensification of dysfunction symptoms and restricted condylar path was observed in patients with the largest partial edentulism and significant level of tooth wear of the residual teeth. A significantly higher percentage of asymptomatic subjects or those with minor dysfunction were also found among the patients with lowest-range partial edentulism in lateral sections.[29]

\section{Effect of Replacement of missing teeth on TMJ}

Temporomandibular joint dysfunction is multifactorial and hence always a multidisciplinary approach is undertaken as the treatment modality. There are few studies that have focused on the relationship between prosthetic rehabilitation and TMJ changes. A study was conducted to evaluate the prevalence of missing mandibular posterior teeth and intraarticular TMDs in a mixed population of asymptomatic subjects and symptomatic TMD patients. The number of missing mandibular bicuspid and molar teeth (excluding third molars) in each subject was recorded, and magnetic resonance images were made to document the presence or absence of disk displacement in the temporomandibular joints. It was concluded that replacement of missing posterior teeth not necessarily prevents the development of TMDs. However, missing mandibular posterior teeth may accelerate the development of degenerative joint disease.[15)]Another study investigated the condyle position in temporal fossa of completely and partially dentate patients pre and post prosthetic treatment, and compares between right and left condyles in both groups by radiographic examination. The results showed that in completely dentate patient, $76.5 \%$ had condyles in concentric position, patients who had lost posterior teeth support showed a predominance of posterior condylar positions and significant decreased in displacement after prosthodontic treatment $(\mathrm{P}=0.015$ for right side and $\mathrm{P}=0.032$ for left side) at significant level. It was documented that significant change in the condylar position occurred after prosthetic rehabilitation, patient's comfort obtained when the position of condyle in glenoid fossa is (concentric position $+0.35 \mathrm{~mm}$ ). The loss of teeth caused remodeling of articular eminence inclination. [30]. It has been suggested in a study that that free end removable partial denture that compensate for the loss of molars in the mandible did not prevent temporomandibular disorder, but enlarging the posterior support may be beneficial to improve occlusal stability and masticatory function and potential to reduce the amount of articular loading .[31]

\section{Conclusion}

Occlusion has not been determined as the dominant cause of TMD problems. However, the interrelationship between this triad of masticatory system, occlusion and temporomandibular joint cannot be overlooked. The effect of partial/ total edentulism on the TMJ health has not been documented through any long term clinical trials due to the complexity of the working of stomatognatic system. Clinicians and researchers should conduct long term studies on the importance of prosthetic rehabilitation to prevent long term unaccounted hazards, not only in the orofacial region but also on the neighboring joints like the spine.

\section{Acknowledgements}

Author would like to thank Dr. Sanjana Ravi Kumar (MDS, Endodontics) and Dr. Sumeet Gupta( BDS) for their help and contribution in data collection and manuscript editing.

\section{REFERENCES}

[1]. Okeson J.P., Management of temporomandibular disorders and occlusion, Mosby, St Luis, 2003.

[2]. Haley DP, Schiffman EL, Lindgren BR, Anderson Q, Andreasen K. The relationship between clinical and MRI findings in patients with unilateral temporomandibular joint pain. J Am Dent Assoc; 132, 2001, 476-481.

[3]. Temporomandibular disorders. In: de Leeuw R, editor. American Academy of Orofacial Pain. Orofacial pain: guidelines for assessment, diagnosis and management. 4th ed. Chicago: Quintessence; 2008,131.

[4]. Dworkin SF, Massoth DL. Temporomandibular disorders and chronic pain: disease or illness? J Prosthet Dent, $72(1), 1994 ; 29-38$.

[5]. Engel GL. The need for a new medical model: a challenge for biomedicine. Science, 196(4286), $1977 ; 129-136$.

[6]. Armellini D, Fraunhofer JA. The shortened dental arch: A review of the literature. J Prosthet Dent ,92, 2004, $531-535$.

[7]. Witter DJ, Creugers NH, Kreulen CM, et al. Occlusal stability in shortened dental arches. J Dent Res ,80,2001, 432-436.

[8]. Okeson JP. Etiology of functional disturbances in the masticatory system. In: Management of temporomandibular disorders and occlusion. St. Louis: Mosby; 2008, 130-163.

[9]. McNamara JA Jr, Seligman DA, Okeson JP. Occlusion, orthodontic treatment, and temporomandibular disorders: a review. $J$ Orofac Pain, 9(1), 1995, 73-90.

[10]. Badel T, Marotti M, Pavicin IS, Basic-Kes V. Temporomandibular disorders and occlusion. Acta Clin Croat.51(3),2012 ,419-424. 
[11]. Gesch D, Bernhardt O, Kirbschus A. Association of malocclusion and functional occlusion with temporomandibular disorders (TMD) in adults: a systematic review of population-based studies. Quintessence Int. 35(3),2004, 211-221.

[12]. Haralur SB, Saeed Al-Shahrani O. Replacement of missing anterior teeth in a patient with temporomandibular disorder. Case Rep Dent. 2014:393627, 2014.

[13]. GREENE CS. THE ETIOLOGY OF TEMPOROMANDIBULAR DISORDERS: IMPLICATIONS FOR TREATMENT. J OROFAC PAIN 15,2001, 93105 .

[14]. Mohl ND, Zarb GA, Carlsson GE, Rugh JD. (Eds.) (1988) A Textbook of Occlusion, Chicago, Quintessence Publishing Company

[15]. Tallents R H, MacherD J, Kyrkanides S, Katzberg RW, Moss ME. Prevalence of missing posterior teeth and intraarticular temporomandibular disorders. J Prosthet Dent, 87, 2002, 45-50.

[16]. Luder H U. Factors affecting degeneration in human temporomandibular joints as assessed histologically. Eur J Oral Sci 110, 2002,106-113.

[17]. Pullinger, AG, Seligman, DA, Gornbein JA. A multiple logistic regression analysis of the risk and relative odds of temporomandibular disorders as a function of common occlusal features. J Dent Res, 72, 1993, 968-979.

[18]. Hansson LG., Hansson T, Petersson A. A Comparison between clinical and radiological findings in 259 temporomandibular joint patients. J Prosthet Dent,50, 1983, 89-94.

[19]. De Boever JA, Carlsson GE, Klineberg IJ. Need for occlusal therapy and prosthodontic treatment in the management of temporomandibular disorders. Part II: Tooth loss and prosthodontic treatment. J Oral Rehabil, 27,2000, 647-659.

[20]. Mejersjo C, Carlsson GE. Analysis of factors influencing the long term treatment of TMJ-pain dysfunction. J Oral Rehabil, 11, 1984,289-295

[21]. Nilner M. Relationships between oral parafunctions and functional disturbances and diseases of the stomatognathic system among children aged 714 years. Acta Odontol Scand 41,1983,167-172.

[22]. Razook SJ, Gotcher JE Jr, Bays RA. Temporomandibular joint noises in infants review of the literature and report of cases. Oral Surg Oral Med Oral Pathol 67, 1989, 658-661.

[23]. Perry HT Jr. Adolescent temporomandibular joint dysfunction. Am J Orthod 63,1973, 517- 525.

[24]. Nilner M, Lassing SA. Prevalence of functional disturbances and diseases of stomatognathic system in 7-14 year olds. Swed Dent J, 5,1981,173-187.

[25]. Gupta SK, Pratibha PK, Bhat KM,1 Mutalik S, Guddattu V. Non-replaced Mandibular First Molars and Temporomandibular Joint Dysfunction. Nepal Journal of Medical Sciences, 3(1),2014, 58-63.

[26]. Majewski S, Gnatofizjologia stomatologiczna. Normy okluzji i funkcje układu stomatognatycznego, Wydawnictwo PZWL, Warszawa, 2007.

[27]. Okeson JP.Management of temporomandibular disorders and occlusion, Mosby, St Luis, 2003.

[28]. Kossioni AE, Dontas AS. The stomatognathic system in the elderly. Useful information for the medical practitioner, Clin. Interv. Aging.2(4), 2007,591-597.

[29]. Krzemien J, Baron S. Axiographic and clinical assessment of temporomandibular joint function in patients with partial edentulism. Acta of Bioengineering and Biomechanics ,15(1), 2013.

[30]. Hatim NA, Jameel NG, Shehab MM. Temporomandibular Joint Assessment of Pre and Post Prosthetic Treatment of Partially Edentulous Patient (Radiographic Examination). Al-Rafidain Dent J, 11(1), 2011,12-23.

[31]. Witter DJ, De Haan AF, Kayser AF, and Van Rossum GM. A 6- year follow-up study oral function in shorten dental arches. Part II : crainomandibular dysfunc-tion and oral comfort. J Oral Rehabil. 21,1994,353-366. 